\title{
Multi-scale Characterization Study Enabling Deactivation Mechanism in Formed Zeolite Catalyst
}

Qianying Guo ${ }^{1}$, Harry M. Meyer III ${ }^{1}$, Anton Ievlev ${ }^{1}$, Anne Starace ${ }^{2}$, Calvin Mukarakate ${ }^{2}$, Susan E. Habas ${ }^{2}$, Gabriel M. Veith ${ }^{1}$ and Kinga A. Unocic ${ }^{1}$

${ }^{1}$ Oak Ridge National Laboratory, Oak Ridge, Tennessee, United States, ${ }^{2}$ National Renewable Energy Laboratory, Golden, Colorado, United States

Zeolites are widely used as catalysts in numerous industrial processes. One of the deactivation mechanisms of such catalysts is direct blocking of the active sites by carbonaceous deposits, or coke [1]. Therefore, detecting and quantifying the carbon concentration in process relevant formed zeolite catalysts plays an important role in optimizing the catalyst lifetime and informing regeneration [2]. However, sample preparation is challenging to avoid additional carbon incorporation into the porous, inhomogeneous catalyst. Here, we studied large formed ZSM-5 zeolite powder as the catalyst that were exposed to pine vapors during ex situ catalytic fast pyrolysis [3]. For this study, two powders were selected with biomass-to-catalyst (B:C) ratios of 3 and 5, as well as different catalyst temperatures, $500 / 450{ }^{\circ} \mathrm{C}$ for $\mathrm{BC} 3$ and $500 / 550{ }^{\circ} \mathrm{C}$ for $\mathrm{BC} 5$, to ensure coke formation in the samples. Scanning transmission electron microscopy (STEM) imaging and energy dispersive X-ray spectroscopy (EDS) were adopted to characterize the carbon concentration in the spent ZSM-5 powders.

TEM lamella was prepared from the surface of the zeolite powder using a focus ion beam (FIB) in situ lift-out technique. To avoid carbon incorporation during sample preparation, the powder was first sputter coated with nickel $(\sim 200 \mathrm{~nm})$. Such a conductive nickel film also minimized the potential 'sample charging' during the lift-out process. Next, a layer of tungsten was deposited to protect the surface during FIB milling. Consolidated ZSM-5 powders have high porosity so only a small portion of the porous lamella was thinned with the ion beam current $<45 \mathrm{pA}$, leaving the thicker part to act as an additional support during sample transfer.

The morphology and elemental distribution of the spent catalysts were studied using Z-contrast (STEM) imaging and EDS acquired on an FEI F200X Talos operating at $200 \mathrm{kV}$ and equipped with Super-X EDS system (4 silicon-drift detectors (SDD) Bruker XFlash $120 \mathrm{~mm} 2$ with a solid angle of 0.9 Steradian). Figures 1(a) and (e) show low magnification high-angle annular dark-field (HAADF)-STEM images of cross-sections of spent BC5 and BC3 ZSM-5 catalysts, respectively. The zeolite crystals were loosely joined by a silica binder with large areas of porosity. Analysis involved investigation of carbon concertation as a function of distance from the exposed catalyst surface. An example of EDS elemental maps of Si and $\mathrm{C}$ is shown for three selected locations/depths $(\sim 1,5,10 \mu \mathrm{m}$ away from external surface) for each catalyst (Fig. 1b-d and 1f-h). In general, higher concentrations of carbon were noticed at the surfaces of the single zeolite crystals (all the carbon maps), while the silicon was homogeneously distributed. 1D line scan analysis across the selected zeolite crystalline surfaces (marked in Fig. 1) was performed and quantified at each depth of both spent catalysts. From the carbon atomic concentration vs. distance curves (Fig. 2), approximately 14 at.\% carbon concentration was detected at the $1.0 \mathrm{~mm}$ depth in spent BC5 ZSM-5, and its concentration decreased to $\sim 5$ at. $\%$ and $\sim 4$ at. $\%$ at $3.8 \mathrm{~mm}$ depth and at $8.4 \mathrm{~mm}$ depth, respectively. For the spent BC3 ZSM-5 catalyst, a similar trend was observed with a higher concertation of carbon near the exposed surface $(\sim 10$ at.\%) and a drop in the carbon concentration 6 at.\% $(6.6 \mathrm{~mm})$ and $\sim 5$ at.\% $(10.2 \mathrm{~mm})$ with increasing distance from the external catalyst surface. Both results in Figure 2 indicate that coke forms closer to the surface of the ZSM-5 crystals. In the future, more statistical analysis is required to show differences between spent BC5 vs. BC3 ZSM-5 catalyst.

The functionality of zeolite-based catalysts for their expected performance and service life depends on their resistance to coke formation or other degradation process. This study shows that FIB-STEM analysis is reliable technique to study the degradation mechanisms of consolidated commercial zeolite [4]. 

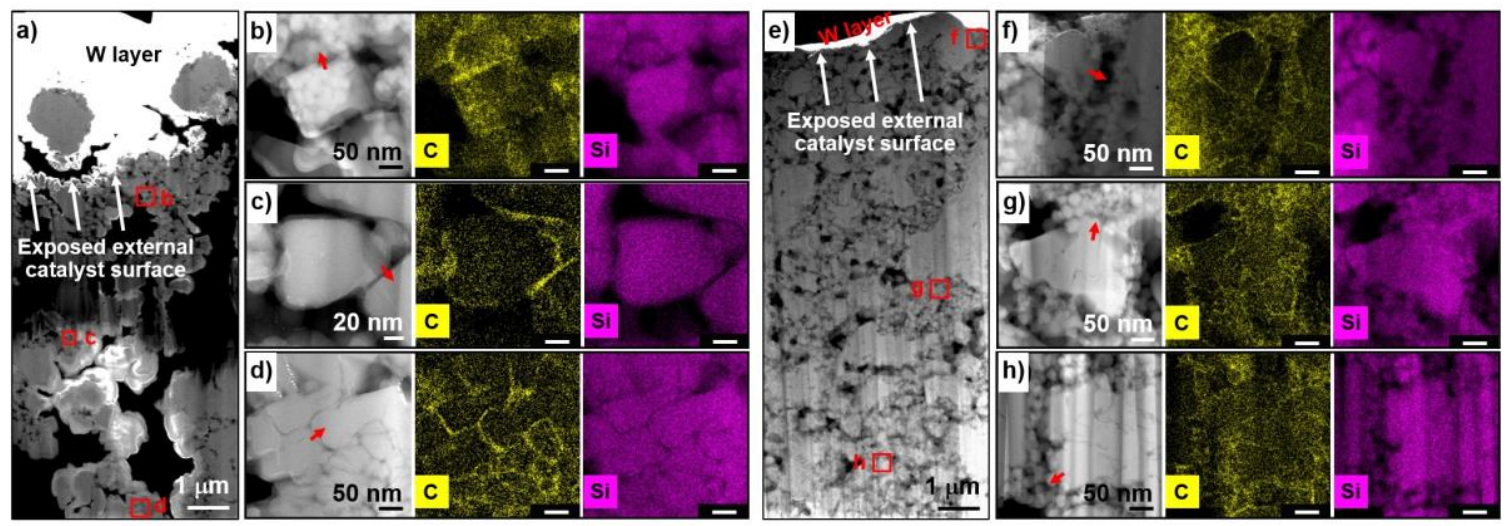

Figure 1. Low magnification HAADF-STEM image of the FIB-prepared spent (a) BC5 and (e) BC3 ZSM-5 powder. Higher magnification HAADF-STEM images and correlated elemental maps generated near and away from the external catalyst surface are shown in (b) (c) (d) and (f) (g) (h) and are marked in (a) and (e), respectively.

(a)

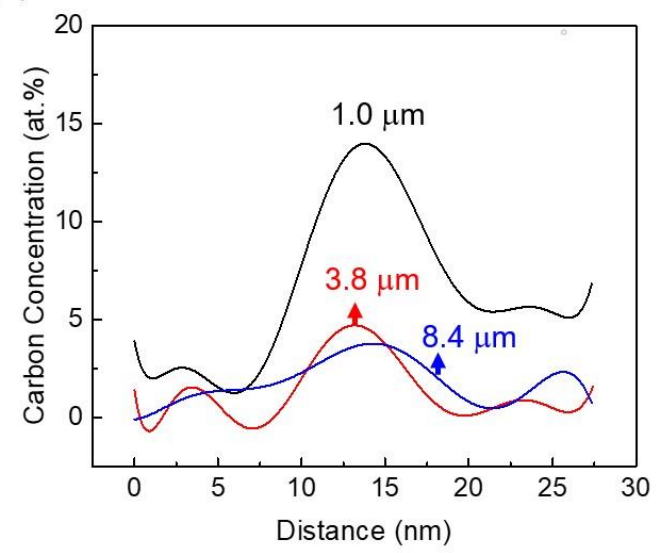

(b)

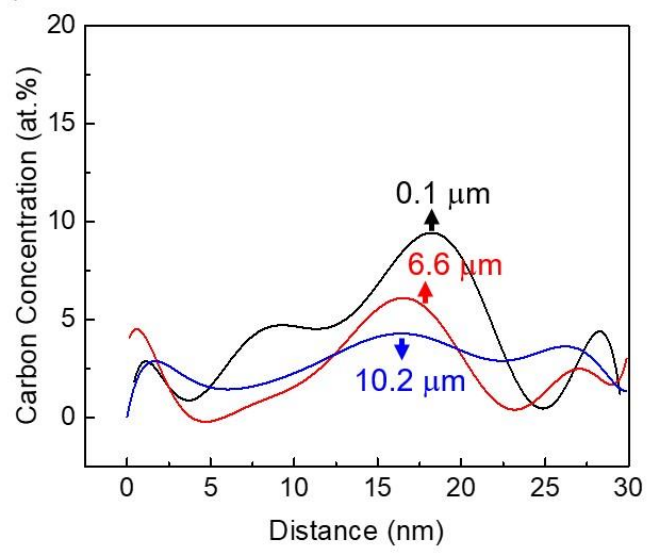

Figure 2. Fitted carbon atomic concentration vs. distance curves generated at different depth from spent (a) BC5 and (b) BC3 ZSM-5. Fitted lines are along the arrows marked in Figure 1.

\section{References}

[1] B. Guido, et al., Chem. Rev. 107 (2007), p. 5366-5410

[2] W.E. Farneth, et al., Chem. Rev. 95 (1995), p. 615-635.

[3] A. Aho, et al., Fuel 87 (2008), p. 2493-2501.

[4] Funding was provided as part of the ChemCatBio Consortium, an Energy Materials Network consortium funded by the U.S. DOE, Office of Energy Efficiency and Renewable Energy, Bioenergy Technologies Office. Microscopy conducted using instruments that are part of (1) ORNL Nuclear Science User Facilities d (2) ORNL Center for Nanophase Materials Sciences (CNMS), which is a U.S. DOE Office of Science User Facility. 